\title{
Reproductive strategies and genetic variability in tropical freshwater fish
}

\author{
Maria Dolores Peres Lassala and Erasmo Renesto \\ Núcleo de Pesquisas em Limnologia, Ictiologia e Aqüicultura, Universidade Estadual de Maringá, \\ Maringá, PR, Brazil.
}

\begin{abstract}
We estimated the genetic variability of nine fish species from the Brazilian upper Paraná River floodplain (Astyanax altiparanae, Hoplias malabaricus, Leporinus lacustris, Loricariichthys platymetopon, Parauchenipterus galeatus, Pimelodus maculatus, Rhaphiodon vulpinus, Roeboides paranensis and Serrasalmus marginatus) based on data for 36 putative allozyme loci obtained using corn starch gel electrophoresis of 13 enzymatic systems: aspartate aminotransferase (EC 2.6.1.1), acid phosphatase (EC 3.1.3.2), esterase (EC 3.1.1.1), glycerol-3-phosphate dehydrogenase (EC 1.1.1.8), glucose-6-phosphate dehydrogenase (EC 1.1.1.49), glucose-6-phosphate isomerase (EC 5.3.1.9), Iditol dehydrogenase (EC 1.1.1.14), isocitrate dehydrogenase - $\mathrm{NADP}^{+}(\mathrm{EC}$ 1.1.1.42), L-lactate dehydrogenase (EC 1.1.1.27), malate dehydrogenase (EC 1.1.1.37), malate dehydrogenase-NADP ${ }^{+}$(EC 1.1.1.40), phosphoglucomutase (EC 5.4.2.2) and superoxide dismutase, (EC 1.15.1.1). The mean expected heterozygosity varied from zero to 0.147 . When data from the literature for 75 species of tropical fish were added to the nine species of this study, the heterozygosity values differed significantly among the groups of different reproductive strategies. The highest mean heterozygosity was for the non-migratory without parental care, followed by the long-distance migratory, and the lowest mean was for the non-migratory with parental care or internal fecundation.
\end{abstract}

Key words: genetic variability, heterozygosity, Paraná River, polymorphism, reproductive strategies, tropical fish.

Received: July 19, 2006; Accepted: March 16, 2007.

\section{Introduction}

The reactions that species offer to environmental selective pressures is reflected in their physiological strategies, which can reduce maintenance energy, increase the efficiency of energy acquisition and, consequently, optimize reproductive efficiency. The success of such strategies may be estimated by the individual ability of being genetically present in the offspring (Agostinho and Júlio Jr., 1999).

In fish, reproductive strategies are extremely diversified, encompassing variations in the way they meet and attract partners, in spawning locations and parental care (Wootton, 1990). External fertilization and embryonic development are the dominant pattern for the majority of teleost fish, although internal fertilization and external embryonic development occurs in some Brazilian freshwater species such as Parauchenipterus galeatus. Blumer (1982) described parental care as any type of investment that enhances the survival probability of offspring (reproductive success) and which vary from pre-fertilization ac-

Send correspondence to Erasmo Renesto. Núcleo de Pesquisas em Limnologia, Ictiologia e Aqüicultura, Universidade Estadual de Maringá, Av. Colombo 5790, 87020-900 Maringá, PR, Brazil. E-mail: erenesto@ hotmail.com. tivities such as nest building (e.g. in Hoplias malabaricus) to mouth brooding and live-bearing.

Adaptation to heterogeneous environments depends upon the genetic variability of species, reduction in genetic variability resulting in reduced feeding and reproductive performance leading to inefficient exploration of the habitats (Kirpichnikov, 1992). Several authors have tried to explain the mechanisms which maintain genetic variability in natural populations. Nevo (1988) analyzed the relationship between heterozygosity and ecological and biological features such as climate (arctic, temperate and tropical), geographic range (wide, narrow, endemic), habitat type (underground, terrestrial, aquatic), habitat range (specialist, generalist) aridity (arid, sub-arid, sub-humid, mesic with a moderate or well-balanced moisture supply) and territoriality (territorial, non-territorial) in 1,111 species (vertebrates, invertebrates, and plants) and concluded that environmental heterogeneity is the major factor in maintaining and structuring genetic diversity in natural populations. Ward et al. (1994) compared the heterozygosity of marine, freshwater and anadromous (fish which live mostly in the sea but breed in fresh water) fish and concluded that marine fish have greater genetic variability than the other types. Heithaus and Laushman (1997) investigated the effects of ecology, life history and water quality on genetic variation 
of three stream-dwelling fish species and revealed that the genetic variability tends to decrease as species become more ecologically specialized.

Allozyme electrophoresis has been extensively used to estimate genetic variability in natural populations (Nevo, 1988; Van Der Bank et al., 1989; Ward et al., 1994) and our study employed this technique to estimate the genetic variability of nine species of fish from the Brazilian upper Paraná River floodplain and, jointly with literature data, verify the relationships between genetic variability and reproductive strategies.

\section{Material and Methods}

From March to December, 2002 in the Brazilian upper Paraná River floodplain, nine species from the most abundant fish with different reproductive strategies were sampled from Baía River (22 $\left.44^{\prime} \mathrm{S}, 53^{\circ} 17^{\prime} \mathrm{W}\right)$ and Paraná River (22 $46^{\prime} \mathrm{S}, 53^{\circ} 15^{\prime} 22^{\prime}$ 'W) (Figure 1). The species sampled were Astyanax altiparanae Garutti \& Britski 2000, Hoplias malabaricus (Bloch 1794), Leporinus lacustris Campos 1945, Loricariichthys platymetopon Isbrücker \& Nijssen 1979, Parauchenipterus galeatus (Linnaeus 1766), Pimelodus maculatus Lacépède 1803, Rhaphiodon vulpinus Agassiz 1829, Roeboides paranensis Pignalberi 1975 and Serrasalmus marginatus Valenciennes 1847. A total of 267 specimens were sampled.

Immediately after capture, white skeletal muscle, gill, heart and liver tissue were removed from each specimen and frozen in liquid nitrogen. Tissues were homogenized with

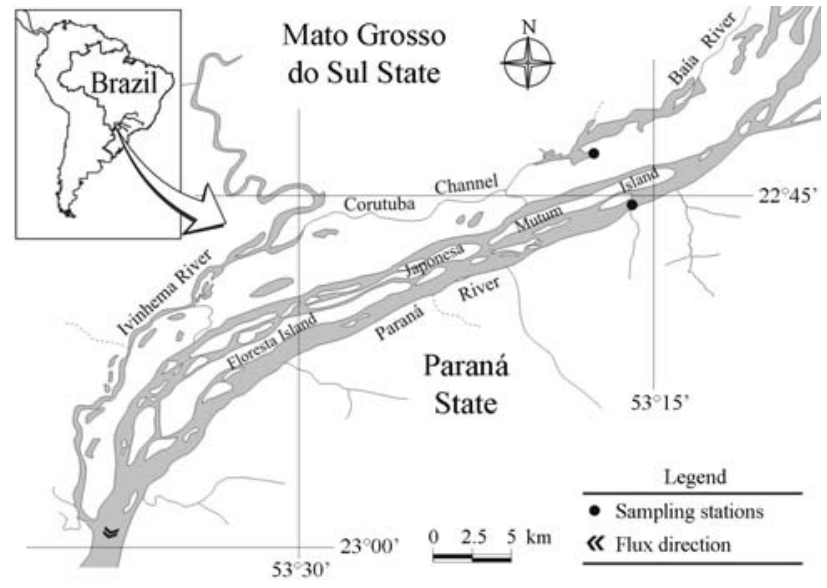

Figure 1 - Paraná River floodplain. Sample localities are indicated by a black circle.

plastic sticks in $1.5 \mathrm{~mL}$ microcentrifuge tubes containing Tris/0.02 M HCl (1:1 w:v) buffer, $\mathrm{pH}$ 7.5. Carbon tetrachloride $\left(\mathrm{CCl}_{4}\right)$ was added to the homogenized liver samples $(1: 2$ $\mathrm{v}: \mathrm{v})$ due to the large amounts of fat present in the tissues (Pasteur et al., 1988). The homogenized samples were centrifuged at $45,114 \mathrm{x} \mathrm{g}$ for $30 \mathrm{~min}$ at temperatures between $1^{\circ}$ and $5{ }^{\circ} \mathrm{C}$ and the supernatants submitted to horizontal electrophoresis in 15\% corn starch gel (Val et al., 1981).

We evaluated 16 enzymatic systems (Table 1), enzyme nomenclature following the proposals of Murphy et al. (1996). Electrophoreses conditions were according to the following authors: Boyer et al., (1963) for SOD; Ruvolo-Takasusuki et al., (2002) for ACP and EST; Shaw

Table 1 - Electrophoretic conditions for 16 enzymatic systems analyzed for species from the upper Paraná River floodplain. The enzymes were run for $14 \mathrm{~h}$, except for $\alpha$ - esterase (EST) EC 3.1.1.1 and acid phosphatase (ACP) EC 3.1.3.2, which were both run for $5.5 \mathrm{~h}$.

\begin{tabular}{|c|c|c|}
\hline Enzymes (abbreviation) and enzyme commission number & Tissue & Buffer* \\
\hline Aspartate aminotransferase (AAT) EC 2.6.1.1 & Liver & I \\
\hline Alcohol dehydrogenase (ADH) EC 1.1.1.1 & Liver & II \\
\hline$\alpha$ - Esterase (EST) EC 3.1.1.1 & Liver & III \\
\hline Acid phosphatase (ACP) EC 3.1.3.2 & Liver & III \\
\hline Glucose dehydrogenase (GCDH) EC 1.1.1.118 & Liver & IV \\
\hline Glycerol-3-phosphate dehydrogenase (G3PDH) EC 1.1.1.8 & Liver & IV \\
\hline Glucose-6-phosphate isomerase (GPI) EC 5.3.1.9 & Heart, liver and muscle & IV \\
\hline Glucose-6-phosphate dehydrogenase (G6PDH) EC 1.1.1.49 & Liver & I \\
\hline L-Iditol dehydrogenase (L-IDDH) EC 1.1.1.14 & Liver & IV \\
\hline Isocitrate dehydrogenase -NADP ${ }^{+}$(IDHP) EC 1.1.1.42 & Heart, gill and liver & IV \\
\hline L-Lactate dehydrogenase (LDH) EC 1.1.1.27 & Heart & IV \\
\hline Malate dehydrogenase $-\mathrm{NAD}^{+}(\mathrm{MDH}) \mathrm{EC}$ 1.1.1.37 & Gill, heart, liver and muscle & IV \\
\hline Malate dehydrogenase -NADP ${ }^{+}$(MDHP) EC 1.1.1.40 & Heart and muscle & IV \\
\hline Peroxidase (PER) EC 1.11.1.7 & Liver & IV \\
\hline Phosphoglucomutase (PGM) EC 5.4.2.2 & Liver and heart & IV \\
\hline Superoxide dismutase (SOD) EC 1.15.1.1 & Liver & II \\
\hline
\end{tabular}

*I = Tris/EDTA/Maleate pH 7.4 (Shaw \& Prasad, 1970); II = Tris/EDTA/borate pH 8.6 (Boyer et al., 1963); III = Tris-HCl pH 7.5 (Ruvolo-Takasusuki et al., 2002); IV = Tris/citrate pH 7.0 (Shaw and Prasad, 1970). 
and Prasad (1970) for AAT, G3PDH, G6PDH, GPI, IDDH, IDHP, LDH, MDH, MDHP and PGM. Standard histochemical staining procedures were used to visualize specific enzymes (Aebersold et al., 1987). Genetic interpretation of the gels was based on the quaternary structure of the enzymes (Ward et al., 1992). Data were analyzed using the POPGENE program version 1.31 (Yeh et al., 1997). Genetic variability was estimated using Nei's unbiased heterozygosity (He) or gene diversity (Nei, 1978). The observed $(\mathrm{Ho})$ and expected $(\mathrm{He})$ heterozygosities for each putative loci and the overall loci means were also calculated. Genotypic frequencies were tested for Hardy-Weinberg equilibrium using the chi squared $\left(\chi^{2}\right)$ test.

The species were organized in three distinct groups of reproductive strategy according to Vazzoler and Menezes (1992): group 1 (G1), containing the long-distance (>100 km) migratory species P. maculatus and $R$. vulpinus; group 2 (G2), containing the sedentary or short-distance $(<100 \mathrm{~km})$ migratory species A. altiparanae, L. lacustris, $R$. paranensis which provide no parental care; and group 3, containing the sedentary or short-distance migratory species $H$. malabaricus, L. platymetopon, P. galeatus, S. marginatus with internal fertilization or which provide parental care. Since it is unlikely that the species analyzed by us were representative of all tropical fish, we added data from the literature for a further 75 tropical fish species (61 from Brazil and 14 from Africa) for which the heterozygosity values had been estimated using allozyme data (Table 2), hence the total number of species analyzed was 84 .

The significance of differences between groups was evaluated using a null models analysis of variance and the EcoSim 7 program (Gotelli \& Entsminger, 2006).

\section{Results}

\section{Genetic variability}

The calculated genetic variability values for each species are presented in Table 3, from which it can be seen that the greatest frequency of polymorphic loci, average number of alleles per locus and heterozygosity were obtained for A. altiparanae, followed by H. malabaricus. No polymorphic locus was detected in R. vulpinus for the 29 loci analyzed. Except for P. maculatus the expected heterozygosity were higher than obtained heterozygosity, which indicates, on average, an excess of homozygotes for all loci.

All loci were in Hardy-Weinberg equilibrium (HWE) for $L$. lacustris, L. platymetopon, P. maculatus, $R$. vulpinus and $R$. paranensis. The loci not in HWE were G6pdh-1, Iddh-1 and Mdhp-1 for A. altiparanae, Gdh-1 for $S$. marginatus, Gdh-2 for P. galeatus, Adh-1, G6pdh-1, Gpi-A, Gpi-b, sMdh-B and Sod-1 for H. malabaricus.

\section{Heterozygosity and reproductive strategies}

The lowest heterozygosity values in our sample of nine species were for the G1 long-distance migratory species $(R$. vulpinus $=0$ and $P$. maculatus $=0.011)$, which therefore also presented the lowest group average $(\mathrm{He}=0.005)$. The highest average heterozygosity $(\mathrm{He}=0.110)$ occurred in the G2 sedentary or short-distance migratory species without parental care, with $A$. altiparane having the highest heterozygosity $(0.152)$ of all the species analyzed. The G3 sedentary or short-distance migratory species with internal fertilization or parental care exhibited intermediate heterozygosity (0.067).

When we considered the nine tropical fish species analyzed by us plus the 75 species from the other studies we found that the number of species in each reproductive strategy group and the average heterozygosity $(\mathrm{He}) \pm$ the standard error for each group was as follows: $\mathrm{G} 1=7$ $(\mathrm{Ho}=0.064 \pm 0.021), \mathrm{G} 2=8(\mathrm{He}=0.081 \pm 0.016)$ and $\mathrm{G} 3=69(\mathrm{He}=0.046 \pm 0.002)$ (Table 3, Figure 2), with $\mathrm{He}$ varying from zero to 0.142 for $\mathrm{G} 1$, from 0.006 to 0.152 for G2, and from zero to 0.143 for G3 (Tables 2 and 3). These data show that the highest average heterozygosity was presented by the G2 sedentary or short-distance migratory species without parental care, the G1 long-distance migratory species had and intermediate average heterozygosity and the G3 species with parental care or internal fertilization presented the lowest average heterozygosity.

A Shapiro-Wilk test for normality showed that the $\mathrm{He}$ values were not normally distributed $(\mathrm{W}=0.9199$, $p<0.0001)$ and the Levene test for homogeneity of variances showed a significant value $(\mathrm{F}=4.843, \mathrm{p}=0.0103)$, indicating that the variances were heterogeneous. We conducted ANOVA but since the assumptions of normality and homogeneity of variances were not met by the data the significance of the ANOVA was tested by null models using the EcoSim 7 programs. The ANOVA detected a significant difference $\left(\mathrm{F}_{2,81}=4.02 ; \mathrm{p}<0.05\right)$ between the three reproductive strategy groups.

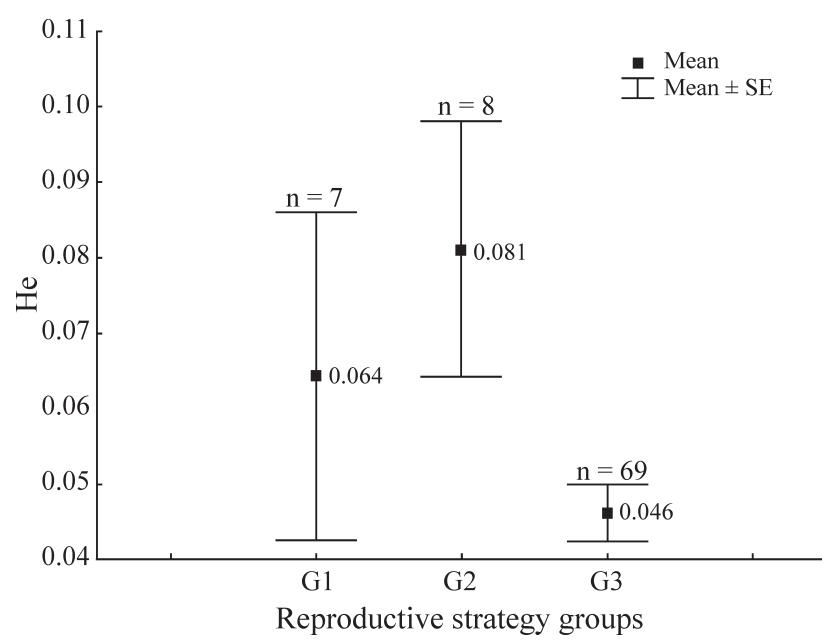

Figure 2 - Mean heterozygosity values for each reproductive strategy group of tropical fish species (Brazil and Africa). Group $1(\mathrm{G} 1)=$ migratory species; Group $2(\mathrm{G} 2)=$ short-distance migratory or sedentary species without parental care; and Group $3(\mathrm{G} 3)=$ short-distance migratory or sedentary species with parental care or internal fertilization. 
Table 2 - Heterozygosity $(\mathrm{He})$ values estimated for 75 tropical fish species from Brazil and Africa based on enzymatic data. Group $1=$ Migratory species, Group 2 = sedentary species or short-distance migratory without parental care; Group 3 = sedentary species or short-distance migratory with internal fertilization or parental care. Africa $=$ Af, Brazil $=$ Br.

\begin{tabular}{|c|c|c|c|}
\hline $\begin{array}{l}\text { Reproductive strategy group, species } \\
\text { (country of isolation, reference*) }\end{array}$ & Heterozygosity & $\begin{array}{l}\text { Reproductive strategy group, species } \\
\text { (country of isolation, reference*) }\end{array}$ & Heterozygosity \\
\hline Group 1 & & Group3 (continued) & \\
\hline Leporinus elongatus $(\mathrm{Br}, 12)$ & 0.142 & Hypostomus sp1 (Br, 4) & 0.042 \\
\hline Leporinus obtusidens $(\mathrm{Br}, 12)$ & 0.090 & Hypostomus sp1 (Br, 9) & 0.028 \\
\hline Pinirampu pirinampus $(\mathrm{Br}, 11)$ & 0.043 & Hypostomus sp1 (Br, 5) & 0.107 \\
\hline Prochilodus lineatus $(\mathrm{Br}, 16)$ & 0.132 & Hypostomus sp1 (Br, 8) & 0.143 \\
\hline \multirow[t]{2}{*}{ Rhinelepis aspera $(\mathrm{Br}, 5)$} & 0.032 & Hypostomus sp1 (Br, 6) & 0.060 \\
\hline & & Hypostomus sp2 (Br, 9) & 0 \\
\hline Group 2 & & Hypostomus sp2 (Br, 8) & 0.059 \\
\hline Iheringichthys labrosus $(\mathrm{Br}, 11)$ & 0.083 & Hypostomus sp2 (Br, 5) & 0.062 \\
\hline Leporinus friederici $(\mathrm{Br}, 12)$ & 0.132 & Hypostomus sp2 (Br, 4) & 0.009 \\
\hline Pimelodus ortimani $(\mathrm{Br}, 15)$ & 0.024 & Hypostomus sp2 (Br, 6) & 0.072 \\
\hline Pimelodus sp (Br, 15) & 0.006 & Hypostomus $\mathrm{sp} 3(\mathrm{Br}, 5)$ & 0.048 \\
\hline \multirow[t]{2}{*}{ Schizodon nasutus $(\mathrm{Br}, 12)$} & 0.092 & Hypostomus sp3 (Br, 8) & 0.091 \\
\hline & & Hypostomus sp3 (Br, 6) & 0.053 \\
\hline Group 3 & & Hypostomus sp3 (Br, 4) & 0.032 \\
\hline Chetia flaviventris (Af, 1) & 0.015 & Hypostomus $\mathrm{sp} 4(\mathrm{Br}, 4)$ & 0.080 \\
\hline Clarias gariepinus (Af, 2) & 0.042 & Hypostomus sp5 (Br, 4) & 0.099 \\
\hline Crenicichla iguassuensis $(\mathrm{Br}, 3)$ & 0.033 & Hypostomus sp6 (Br, 4) & 0.021 \\
\hline Crenicichla $\mathrm{sp}(\mathrm{Br}, 3)$ & 0.063 & Hypostomus sp7 (Br, 4) & 0.063 \\
\hline Hemichromis elongatus (Af, 1) & 0.020 & Hypostomus ternetzi $(\mathrm{Br}, 5)$ & 0.069 \\
\hline Hisonotus sp $(\mathrm{Br}, 4)$ & 0 & Loricariichthys anus $(\mathrm{Br}, 13)$ & 0.023 \\
\hline Hypostomus albopunctatus $(\mathrm{Br}, 5)$ & 0.031 & Loricariichthys platymetopon $(\mathrm{Br}, 13)$ & 0.050 \\
\hline Hypostomus ancistroides $(\mathrm{Br}, 5)$ & 0.082 & Loricariichthys $\mathrm{sp}(\mathrm{Br}, 13)$ & 0.035 \\
\hline Hypostomus ancistroides $(\mathrm{Br}, 4)$ & 0.052 & Megalancistrus parananus $(\mathrm{Br}, 5)$ & 0.019 \\
\hline Hypostomus boulengeri $(\mathrm{Br}, 6)$ & 0.078 & Neoplecostomus paranensis $(\mathrm{Br}, 14)$ & 0 \\
\hline Hypostomus cochliodon $(\mathrm{Br}, 6)$ & 0.070 & Neoplecostomus sp $(\mathrm{Br}, 14)$ & 0.030 \\
\hline Hypostomus cochliodon $(\mathrm{Br}, 5)$ & 0.039 & Oreochromis andersonii (Af, 1) & 0.019 \\
\hline Hypostomus aff commersoni $(\mathrm{Br}, 7)$ & 0.044 & Oreochromis macrochir (Af, 1) & 0.031 \\
\hline Hypostomus commersoni $(\mathrm{Br}, 5)$ & 0.088 & Pterygoplichthys anisitsi $(\mathrm{Br}, 5)$ & 0.032 \\
\hline Hypostomus aff derbyi $(\mathrm{Br}, 5)$ & 0.037 & Pterygoplichthys anisitsi $(\mathrm{Br}, 6)$ & 0.038 \\
\hline Hypostomus derbyi $(\mathrm{Br}, 7)$ & 0.011 & Serranochromis angusticeps (Af, 1) & 0.013 \\
\hline Hypostomus hemanni $(\mathrm{Br}, 8)$ & 0.068 & Serranochromis carlottae (Af, 1$)$ & 0.031 \\
\hline Hypostomus $\mathrm{cf}$ latirostris $(\mathrm{Br}, 6)$ & 0.029 & Serranochromis condringtonii (Af, 1) & 0.035 \\
\hline Hypostomus latifrons $(\mathrm{Br}, 6)$ & 0.070 & Serranochromis giardi (Af, 1) & 0.013 \\
\hline Hypostomus microstomus $(\mathrm{Br}, 5)$ & 0.023 & Serranochromis macrocephalus (Af, 1) & 0.021 \\
\hline Hypostomus margaritifer $(\mathrm{Br}, 10)$ & 0.061 & Serranochromis robustus (Af, 1) & 0.018 \\
\hline Hypostomus margaritifer $(\mathrm{Br}, 5)$ & 0.106 & Serranochromis thumbergi (Af, 1) & 0.018 \\
\hline Hypostomus myersi $(\mathrm{Br}, 7)$ & 0.017 & Tilapia rendalli (Af, 1) & 0.031 \\
\hline Hypostomus paulinus $(\mathrm{Br}, 9)$ & 0.027 & Tilapia sparrmanii $(\mathrm{Af}, 1)$ & 0.032 \\
\hline Hypostomus regani $(\mathrm{Br}, 6)$ & 0.033 & & \\
\hline Hypostomus regani $(\mathrm{Br}, 4)$ & 0.056 & & \\
\hline Hypostomus regani $(\mathrm{Br}, 5)$ & 0.098 & & \\
\hline
\end{tabular}

*References (see reference list for full references): 1. Van der Bank et al. (1989), 2. Van der Bank et al. (1992), 3. Renesto et al. (2001), 4. Zawadzki \& Renesto (unpublished data), 5. Zawadzki et al. (2005), 6. Renesto E. (unpublished data), 7. Zawadzki et al. (1999), 8. Zawadzki et al. (2004)a, 9. Paiva et al. (2005), 10. Zawadzki et al. (2002), 11. Almeida \& Sodré (1998), 12. Chiari \& Sodré (1999), 13. Zawadzki et al. (2000), 14. Zawadzki et al. (2004)b, 15. Renesto et al. (2000), 16. Revaldaves et al. (1997). 
Table 3 - Allele frequencies at 36 loci of nine fish species from the Paraná River floodplain. Bold type numbers are loci not in Hardy-Weinberg equilibrium. The nine species were: Astyanax altiparanae (Apar), Leporinus lacustris (Lcut), Loricariichthys platymetopon (Lpty), Pimelodus maculatus (Pmct), Rhaphiodon vulpinus (Rvlp), Roeboides paranensis (Rprn), Serrasalmus marginatus (Smgt), Parauchenipterus galeatus (Pglt) and Hoplias malabaricus (Hmal). Numbers in boldface type indicate the loci which are not in Hardy-Weinberg equilibrium.

\begin{tabular}{|c|c|c|c|c|c|c|c|c|c|c|}
\hline Locus & Alleles & Apar & Lcut & Lpty & Pmct & Rvlp & Rprn & Smgt & Pglt & Hmal \\
\hline Aat-1 & $\begin{array}{l}a \\
b\end{array}$ & 1.000 & 1.000 & 1.000 & 1.000 & 1.000 & $\begin{array}{l}0.965 \\
0.035\end{array}$ & 1.000 & 1.000 & 1.000 \\
\hline Aat-2 & $\begin{array}{l}a \\
b\end{array}$ & & & & 1.000 & 1.000 & 1.000 & $\begin{array}{l}0.033 \\
0.967\end{array}$ & 1.000 & 1.000 \\
\hline Acp-1 & $\begin{array}{l}a \\
b \\
c\end{array}$ & 1.000 & 1.000 & 1.000 & 1.000 & 1.000 & $\begin{array}{l}0.967 \\
0.033\end{array}$ & 1.000 & 1.000 & 1.000 \\
\hline Acp-2 & $a$ & & 1.000 & & & 1.000 & & & 1.000 & \\
\hline Adh-1 & $\begin{array}{l}a \\
b\end{array}$ & & 1.000 & 1.000 & 1.000 & 1.000 & $\begin{array}{l}0.200 \\
0.800\end{array}$ & & 1.000 & $\begin{array}{l}0.729 \\
0.271\end{array}$ \\
\hline$A d h-2$ & $a$ & & & & & & & & 1.000 & \\
\hline Est-1 & $\begin{array}{l}a \\
b \\
c\end{array}$ & $\begin{array}{l}0.065 \\
0.903 \\
0.032\end{array}$ & 1.000 & 1.000 & 1.000 & 1.000 & $\begin{array}{l}0.817 \\
0.150 \\
0.033\end{array}$ & $\begin{array}{l}0.017 \\
0.766 \\
0.217\end{array}$ & 1.000 & $\begin{array}{l}0.090 \\
0.455 \\
0.455\end{array}$ \\
\hline Est-2 & $\begin{array}{l}a \\
b \\
c\end{array}$ & $\begin{array}{l}0.984 \\
0.016\end{array}$ & $\begin{array}{l}0.683 \\
0.300 \\
0.017\end{array}$ & 1.000 & 1.000 & 1.000 & 1.000 & 1.000 & 1.000 & \\
\hline Est-3 & $\begin{array}{l}a \\
b\end{array}$ & & 1.000 & 1.000 & 1.000 & 1.000 & & 1.000 & $\begin{array}{l}0.783 \\
0.217\end{array}$ & \\
\hline Est-4 & $\begin{array}{l}a \\
b\end{array}$ & & & & $\begin{array}{l}0.983 \\
0.017\end{array}$ & & & & & \\
\hline Gdh-1 & $\begin{array}{l}a \\
b \\
c\end{array}$ & & 1.000 & $\begin{array}{l}0.167 \\
0.833\end{array}$ & $\begin{array}{l}0.033 \\
0.967\end{array}$ & 1.000 & 1.000 & $\begin{array}{l}0.034 \\
0.828 \\
0.138\end{array}$ & 1.000 & 1.000 \\
\hline$G d h-2$ & $\begin{array}{l}a \\
b \\
c\end{array}$ & & 1.000 & & & 1.000 & & & $\begin{array}{l}0.200 \\
0.767 \\
0.033\end{array}$ & \\
\hline G3pdh-1 & $\begin{array}{l}a \\
b\end{array}$ & $\begin{array}{l}0.919 \\
0.081\end{array}$ & 1.000 & 1.000 & 1.000 & 1.000 & 1.000 & 1.000 & 1.000 & \\
\hline G3pdh-2 & $\begin{array}{l}a \\
b\end{array}$ & 1.000 & $\begin{array}{l}0.967 \\
0.033\end{array}$ & 1.000 & 1.000 & & & & 1.000 & \\
\hline G6pdh-1 & $\begin{array}{l}a \\
b \\
c\end{array}$ & $\begin{array}{l}0.134 \\
0.775 \\
0.091\end{array}$ & 1.000 & 1.000 & 1.000 & 1.000 & 1.000 & 1.000 & 1.000 & $\begin{array}{l}0.833 \\
0.167\end{array}$ \\
\hline G6pdh-2 & $a$ & & 1.000 & & & 1.000 & & & & \\
\hline$G p i-A$ & $\begin{array}{l}a \\
b \\
c \\
d \\
e \\
f\end{array}$ & $\begin{array}{l}0.113 \\
0.339 \\
0.161 \\
0.258 \\
0.097 \\
0.032\end{array}$ & $\begin{array}{l}0.983 \\
0.017\end{array}$ & 1.000 & $\begin{array}{l}0.933 \\
0.067\end{array}$ & 1.000 & 1.000 & 1.000 & $\begin{array}{l}0.950 \\
0.050\end{array}$ & $\begin{array}{l}0.750 \\
0.250\end{array}$ \\
\hline Gpi-B & $\begin{array}{l}a \\
b \\
c\end{array}$ & $\begin{array}{l}0.968 \\
0.032\end{array}$ & $\begin{array}{l}0.367 \\
0.617 \\
0.017\end{array}$ & $\begin{array}{l}0.850 \\
0.117 \\
0.033\end{array}$ & 1.000 & 1.000 & $\begin{array}{l}0.850 \\
0.133 \\
0.017\end{array}$ & $\begin{array}{l}0.983 \\
0.017\end{array}$ & 1.000 & $\begin{array}{l}0.208 \\
0.479 \\
0.313\end{array}$ \\
\hline$I d d h-1$ & $\begin{array}{l}c \\
a \\
b \\
c\end{array}$ & $\begin{array}{l}0.581 \\
0.339 \\
0.080\end{array}$ & 1.000 & $\begin{array}{l}0.033 \\
1.000\end{array}$ & 1.000 & 1.000 & 0.017 & 1.000 & 1.000 & 0.313 \\
\hline$I d d h-2$ & $a$ & & 1.000 & 1.000 & & & & 1.000 & & \\
\hline Idhp-1 & $\begin{array}{l}a \\
b\end{array}$ & 1.000 & 1.000 & 1.000 & 1.000 & 1.000 & 1.000 & $\begin{array}{l}0.833 \\
0.167\end{array}$ & 1.000 & 1.000 \\
\hline$I d h p-2$ & $\begin{array}{l}a \\
b\end{array}$ & $\begin{array}{l}0.032 \\
0.968\end{array}$ & $\begin{array}{l}0.964 \\
0.036\end{array}$ & 1.000 & 1.000 & 1.000 & $\begin{array}{l}0.933 \\
0.067\end{array}$ & 1.000 & $\begin{array}{l}0.983 \\
0.017\end{array}$ & 1.000 \\
\hline Idhp-3 & & & & & & & & & & 1.000 \\
\hline$L d h-A$ & $a$ & 1.000 & 1.000 & 1.000 & 1.000 & 1.000 & 1.000 & 1.000 & 1.000 & 1.000 \\
\hline$L d h-B$ & $a$ & 1.000 & 1.000 & 1.000 & 1.000 & 1.000 & 1.000 & 1.000 & 1.000 & 1.000 \\
\hline$m M d h-1$ & $\begin{array}{l}a \\
b\end{array}$ & $\begin{array}{l}0.984 \\
0.016\end{array}$ & 1.000 & 1.000 & 1.000 & & & 1.000 & 1.000 & 1.000 \\
\hline
\end{tabular}


Table 3 (cont.)

\begin{tabular}{|c|c|c|c|c|c|c|c|c|c|c|}
\hline Locus & Alleles & Apar & Lcut & Lpty & Pmct & Rvlp & Rprn & Smgt & Pglt & Hmal \\
\hline \multirow[t]{2}{*}{$s M d h-A$} & $a$ & 1.000 & 1.000 & 0.100 & 1.000 & 1.000 & 1.000 & 1.000 & 1.000 & 1.000 \\
\hline & $b$ & & & 0.900 & & & & & & \\
\hline \multirow[t]{2}{*}{$s M d h-B$} & $a$ & 1.000 & 1.000 & 1.000 & 1.000 & 1.000 & 1.000 & 1.000 & 0.983 & 0.604 \\
\hline & $b$ & & & & & & & & 0.017 & 0.396 \\
\hline \multirow[t]{2}{*}{ Mdhp-1 } & $a$ & 0.800 & 0.767 & 1.000 & 1.000 & 1.000 & 0.933 & 1.000 & 1.000 & 0.938 \\
\hline & $b$ & 0.200 & 0.233 & & & & 0.067 & & & 0.063 \\
\hline \multirow[t]{2}{*}{$M d h p-2$} & $a$ & 0.484 & 1.000 & 1.000 & 1.000 & 1.000 & 1.000 & & 1.000 & 1.000 \\
\hline & $b$ & 0.516 & & & & & & & & \\
\hline Per-1 & $a$ & & 1.000 & 1.000 & 1.000 & 1.000 & & 1.000 & 1.000 & 1.000 \\
\hline Per-2 & $a$ & & 1.000 & 1.000 & 1.000 & 1.000 & & & 1.000 & 1.000 \\
\hline Per-3 & $a$ & & & 1.000 & & & & & & \\
\hline \multirow[t]{3}{*}{ Pgm-1 } & $a$ & 1.000 & 0.717 & 1.000 & 0.967 & 1.000 & 0.467 & 1.000 & 0.067 & 1.000 \\
\hline & $b$ & & 0.283 & & 0.033 & & 0.533 & & 0.433 & \\
\hline & $c$ & & & & & & & & 0.500 & \\
\hline \multirow[t]{3}{*}{ Sod-1 } & $a$ & 1.000 & 0.352 & 1.000 & 1.000 & 1.000 & 1.000 & & 0.633 & 0.318 \\
\hline & $b$ & & 0.093 & & & & & & 0.333 & 0.0682 \\
\hline & $c$ & & 0.555 & & & & & & 0.033 & \\
\hline \multirow[t]{2}{*}{ Sod-2 } & $a$ & & & & 0.983 & 1.000 & & & 1.000 & \\
\hline & $b$ & & & & 0.017 & & & & & \\
\hline \multicolumn{2}{|c|}{$\begin{array}{l}\text { Number of specimens } \\
\text { (N) }\end{array}$} & 31 & 30 & 30 & 30 & 42 & 30 & 30 & 30 & 24 \\
\hline \multicolumn{2}{|c|}{$\begin{array}{l}\text { Average number of } \\
\text { alleles per locus }(\mathrm{K})\end{array}$} & 1.8 & 1.3 & 1.1 & 1.2 & 1.0 & 1.4 & 1.3 & 1.3 & 1.4 \\
\hline \multicolumn{2}{|c|}{$\begin{array}{l}\text { polymorphic loci } \\
\text { frequency }\left(\mathrm{P}_{0.99}\right)\end{array}$} & 0.524 & 0.267 & 0.107 & 0.172 & 0 & 0.381 & 0.217 & 0.226 & 0.333 \\
\hline \multicolumn{2}{|c|}{$\begin{array}{l}\text { Average observed } \\
\text { heterozygosity (Ho) }\end{array}$} & 0.068 & 0.062 & 0.006 & 0.011 & 0 & 0.052 & 0.039 & 0.039 & 0.048 \\
\hline \multicolumn{2}{|c|}{$\begin{array}{l}\text { Average expected } \\
\text { heterozygosity }(\mathrm{He})\end{array}$} & 0.152 & 0.081 & 0.026 & 0.011 & 0 & 0.084 & 0.045 & 0.062 & 0.137 \\
\hline \multicolumn{2}{|c|}{ Standard deviation (SD) } & 0.225 & 0.171 & 0.077 & 0.028 & 0 & 0.142 & 0.108 & 0.154 & 0.220 \\
\hline \multicolumn{2}{|c|}{ He standard error (SE) } & 0.041 & 0.031 & 0.014 & 0.005 & 0 & 0.025 & 0.020 & 0.028 & 0.045 \\
\hline
\end{tabular}

\section{Discussion}

The genetic variability estimated in nine fish species from the upper Paraná River floodplain using Nei's gene diversity $(\mathrm{He})$ varied from zero to 0.1518 with an average of 0.066 , very near to the average of 0.051 described for 195 piscine species from several world-wide localities reviewed by Ward et al. (1992).

It has been generally accepted that populations of long-distance migratory species have higher genetic variability than non-migratory species because of their higher gene flow. Although this is true for several taxa (Ward et al., 1992), our data are not in agreement with this hypothesis. In our study, no polymorphic locus was found in a sample of 29 analyzed loci of $R$. vulpinus, a long-distance migratory species. This is surprising, since other cases of absence of genetic variability have been found in two sedentary species, Neoplecostomus paranensis from the upper Paraná River (Zawadzki et al., 2004b) and Hypostomus sp. 2 from the third-order Paraná River tributary Ribeirão Maringá (Paiva et al., 2005). Furthermore, P. maculatus, the other long-distance migratory species analyzed in our study, showed low genetic variability $(\mathrm{He}=0.011)$, con- trasting with the value of $\mathrm{He}=0.132$ estimated for other migratory species such as Prochilodus lineatus (Revaldaves et al., 1997) and Leporinus friderici (Chiari and Sodré, 1999).

The genetic variability estimated by us for $S$. marginatus $(\mathrm{He}=0.045)$ and $P$. galeatus $(\mathrm{He}=0.062)$ were intermediate compared to the average of 49 freshwater fish species $(\mathrm{He}=0,046)$ estimated by Ward et al. (1994). On the other hand, our estimated values for A. altiparanae $(\mathrm{He}=0,152), H$. malabaricus $(\mathrm{He}=0,137), R$. paranensis $(\mathrm{He}=0,084)$ and L. lacustris $(\mathrm{He}=0,081)$ were high compared to the average found by Ward et al. (1994).

In the nine species analyzed by us the average heterozygosity of species with different reproductive strategies was not statistically different when compared amongst each other, which could lead to the conclusion that reproductive strategies are not related to genetic variability. However, our results could have been biased because the group of migratory species analyzed by us had only two species, so to compensate for this we carried out an analysis of our set of nine species combined with data on 75 tropical fish species from the literature. When the data for the 84 
tropical fish species were analyzed, the relationship between heterozygosity and reproductive strategies were changed and there was a significant difference between the reproductive strategies groups (Figure 2). With this analysis, the average heterozygosity values which differed most were those between the G3 parental care group with and the G2 group without parental care. The heterogeneity of the heterozygosity variances between groups may have been due to the number of species analyzed, since the minimum and maximum heterozygosity values were similar among the groups.

The number of surviving offspring is likely to be greater in species with parental care than in species without parental care (Vazzoler, 1996), and species exhibiting parental care tend to be less variable. This can be observed in L. platymetopon, which brood their fry inside the male's mouth and is the most abundant species of the Paraná River floodplain. In species without parental care, few offspring are likely to survive, and a greater genetic variability is important to face environmental challenges.

Nei (1987) pointed out three main factors related to heterozygosity level are the quaternary structure of proteins, the molecular weight of the protein subunit and the species population size. In addition to these factors, we found that parental care is another factor that should be taken into account to maintain gene diversity. However, since a large number of factors influence the amount of genetic variability, we believe that further studies will be necessary to elucidate the relationship between genetic variability and ecological features of fish species.

\section{Acknowledgments}

The authors would like to thank Dr. Horácio Ferreira Júlio Jr. and Gilmar Lassala for helping with the capture and identification of fish, Jaime L. Pereira for drawing the map, Gislaine Iachstel Manetta for personal help and Dr. Luiz Carlos Gomes for statiscal help. We also thank NUPELIA-UEM for material support, and the Brazilian National Counsel of Technological and Scientific Development (Conselho Nacional de Pesquisa - CNPq) and Coordenação de Aperfeiçoamento de Pessoal de Ensino Superior (CAPES) for financial support.

\section{References}

Aebersold PB, Winans GA, Tell DJ, Milner GB and Utter M (1987) Manual for starch gel electroforesis: A method for the detection of genetic variation. NOAA Technical Report NMFS 61:1-17.

Agostinho AA and Júlio HF Jr (1999) Peixes da bacia do alto rio Paraná. In: Lowe-McConnell RH (ed) Estudos Ecológicos de Comunidades de Peixes Tropicais. Editora da Universidade de São Paulo, São Paulo, pp 374-400.

Almeida FS and Sodré LMK (1998) Analysis of genetic variability in three species of Pimelodidae (Ostariophysi, Siluriformes). Genet Mol Biol 21:487-492.
Boyer SH, Fainer DC and Naughton MA (1963) Myoglobin: Inherited structural variation in man. Science 140:1228-1231.

Blumer LS (1982) A bibliography and categorization of bony fishes exhibiting parental care. Zool J Lin Soc 75:1-22.

Chiari L and Sodré LMK (1999) Genetic variability in five species of anostomidae (Ostariophysi, Characiformes). Genet Mol Biol 4:517-523

Heithaus MR and Laushman RH (1997) Genetic variation and conservation of stream fishes: Influence of ecology, life history, and water quality. Can J Fish Aquat Sci 54:1822-1836.

Kirpichnikov VS (1992) Adaptative nature of intrapopulational biochemical polymorphism in fish. J Fish Biol 40:1-16.

Murphy RW, Sites JW, Buth DG Jr and Haufler CH (1996) Proteins: Isozyme electrophoresis. In: Hillis DM, Moritz C and Mable BK (eds) Molecular Systematics. 2nd edition. Sinnauer Associates, Sunderland, pp 51-120.

Nei M (1987) Molecular Evolutionary Genetics. Columbia University Press, New York, 512 pp.

Nei M (1978) Estimation of average heterozygosity and genetic distance from a small number of individuals. Genetics 89:583-590

Nevo E (1988) Genetic diversity in nature. Evol Biol 23:217-246.

Paiva S, Renesto E and Zawadzki CH (2005) Genetic variability of Hypostomus (Teleostei, Loricariidae) from the Ribeirão Maringá, a stream of the Upper Rio Paraná basin, Brazil. Genet Mol Biol 28:370-375.

Pasteur N, Pasteur G, Bonhomme F, Catalan J and BrittonDavidian J (1988) Practical Isozyme Genetics. Ellis Horwood Limited, Chichester, 215 pp.

Renesto E, Zawadzki CH and Revaldaves E (2001) Biochemical taxonomy of Crenicichla (Pisces, Perciformes, Cichlidae) of the Iguaçu river, Brazil. Brazil Arch Biol Technol 44:15-22.

Renesto E, Zawadzki CH and Revaldaves E (2000) Genetic evidence for two species of genus Pimelodus Lacépède, 1803 (Siluriformes, Pimelodidae) in the Iguaçu River (Brazil). Genet Mol Biol 23:809-813.

Revaldaves E, Renesto E and Machado MFPS (1997) Genetic variability of Prochilodus lineatus (Characiformes, Prochilodontidae) in the Upper Paraná River. Rev Brazil Genet 20:381-388.

Ruvolo-Takasusuki MCC, Machado MFPS and Conte H (2002) Esterase-3 polymorphism in the sugarcane borer Diatraea saccharalis. Genet Mol Biol 25:61-64.

Shaw CR and Prasad R (1970) Starch gel electrophoresis of enzymes: A compilation of recipes. Biochem Genet 4:297320.

Val AL, Schwantes AR, Schwantes MLB and Luca PH (1981) Amido hidrolisado de milho como suporte eletroforético. Ciência e Cultura 33:737-741 (Abstract in English).

Van Der Bank FH, Grobler JP and Du Preez HH (1992) A comparative biochemical genetic study of three populations of domesticated and wild African catfish, Clarias gariepinus. Comp Biochem Physiol 101:387-390.

Van Der Bank FH, Grant WS and Ferreira JT (1989) Electrophoretically detectable genetic data for fifteen southern African cichlids. J Fish Biol 34:465-483.

Vazzoler AEA and Menezes NA (1992) Síntese de conhecimentos sobre o comportamento reprodutivo dos Characiformes da América do Sul (Teleostei, Ostariophysi). Rev Bras Biol 52:627-640. 
Vazzoler AEA (1996) Biologia da Reprodução de Peixes Teleósteos: Teoria e Prática. Editora da Universidade Estadual de Maringá, Maringá, 169 pp.

Ward RD, Skibinski DOF and Woodward M (1992) Protein heterozygosity, protein structure and taxonomic differentiation. Evol Biol 26:73-59.

Ward RD, Woodward M and Skibinski DOF (1994) A comparison of genetic diversity levels in marine, freshwater, and anadromous fishes. J Fish Biol 44:213-232.

Wootton RJ (1990) Ecology of Teleost Fishes. Chapman \& Hall, London, 404 pp.

Yeh FC and Boyle TJB (1997) Population genetic analysis of co-dominant and dominant markers and quantitative traits. Belg J Bot 129:156-157.

Zawadzki CH, Renesto E and Bini LM (1999) Allozyme discrimination of three species of the genus Hypostomus Lacépède, 1803 (Osteichthyes, Loricariidae) from the rio Iguaçu basin (Brazil). Rev Suis Zool 106:91-105.

Zawadzki CH, Reis RE and Renesto E (2000) Allozyme discrimination of three species of Loricariichthys (Siluriformes, Loricariidae) from Southern Brazil. Rev Suis Zool 107:1-12.

Zawadzki CH, Weber C, Pavanelli CS and Renesto E (2002) Morphological and biochemical comparison of two allopatric populations of Hypostomus margaritifer (Regan, 1907) (Osteichthyes, Loricariidae) from the upper Paraná River basin, Brazil. Acta Scientiarum 24:499-505.

Zawadzki CH, Renesto E, Paiva S and Lara-Kamei MCS (2004a) Allozyme differentiation of four populations of Hypostomus (Teleostei, Loricariidae) from Ribeirão Keller, a small stream in the upper Rio Paraná basin, Brazil. Genetica 121:251-257.

Zawadzki CH, Alves AL, Renesto E and Oliveira C (2004b) Biochemical evidence of a possible new species of Neoplecostumus (Teleostei, Loricariidae) from the upper Rio Paraná basin, Brazil. Biochem Syst Ecol 32:573-582.

Zawadzki CH, Renesto E, Reis RE, Moura MO and Mateus RP (2005) Allozyme relationships in hypostomines (Teleostei, Loricariidae) from the Itaipu Reservoir, Upper Rio Paraná basin, Brazil. Genetica 123:271-283.

\section{Internet Resource}

Gotelli NJ and Entsminger GL (2006) EcoSim: Null models software for ecology. Version 7. Acquired Intelligence Inc. \& Kesey-Bear. Jericho, VT 05465. http://garyentsminger.com/ ecosim/index.html.

Associate Editor: João S. Morgante 